\title{
External Qigong Therapy for Chronic Orofacial Pain
}

\author{
A Letter to the Editor \\ Journal of Alternative and Complementary Medicine, 2002, 8(5): 532-534.
}

\section{Dear Editor,}

We would like to share the results of an anecdotal pilot trial on qigong therapy for chronic pain with you. As chronic pain patients are increasingly seeking alternatives to Western medicine (Eisenberg et al. 1998), this anecdotal study was designed as an open trial to collect some pilot data without a control. The study was anecdotal without much plan due to the tight schedule of the qigong healer who was visiting the U.S. for the first time in a short notice.

Subjects: 10 chronic orofacial pain patients (all white females, mean age 54 years) from a private dental practice (JJM) participated. Their duration of pain ranged from 2 years to 35 years (mean $=15$ years). Among the 10 patients, four of them meet criteria for Myofascial face pain (MFP), four for MFP and comorbid fibromyalgia, and 7 of them for phantom tooth pain (4 patients meet criteria for multiple disorders). All of them experienced constant or daily pain when they came to the clinic, and most had not heard of qigong before the study.

Intervention: The subject was seated in a treatment room along with the Qigong healer and the translator $(\mathrm{KC})$. Qigong healer administered qi emission for 5 to 10 minutes. This treatment is said to emit qi ("healing energy") to the area of specific pain at a distance of 10-15 $\mathrm{cm}$ from the patient. Occasionally the patient was touched. At times the Qigong master uttered words in Chinese climaxing in a shout. The master spoke no English; the subjects knew no Chinese. 
Outcome Measures: Visual analogue (VAS) pain and mood scales were used before and after the treatment. Verbal anchors were used from 0 "least possible pain" to 100 "worst possible pain." In addition a categorical pain scale was employed (Wallenstein, et al. 1980). The scale consists of eight verbal descriptors ranging from "no pain" to "excruciating." The mood scale was anchored by the "best I could feel and "worst I could feel." A VAS relief 0-to-100 scale was anchored by "no relief of pain" and "complete relief of pain."

Results: All patients reported some degree of relief and all but one reported reduction in pain after the treatment (See Table 1). Among the 10 patients, all of them reported various degrees of pain relief after the single treatment, ranging from 22 to 95 in the 100 VAS relief scale (mean=48.5). When the pre- and post-VAS pain scores were compared, 8 of the 10 patients reported some degree of reduction in pain, ranging from 2 to 40 in reported pain reduction (mean=16.4), one reported no change, and one reported more pain. The eight subjects reported reduction in pain in various degree, ranging from $11 \%$ to $75 \%$. The subject who reported the pain increase from 22 to 43 on the VAS and from "mild" to "moderate" on the categorical scale also experienced a sharp worsening of mood immediately following treatment. She appeared to become anxious and was agitated by the treatment experience. Using a non-parametric statistic to test the difference between positive response (pain reduction) and the negative response (pain increase), in 8 out of 9 trials the patients reported reduction in pain $(p=0.02)$.

However, the other two indicators in the study, the change in general mood and the change in word description of pain, are not entirely consistent with the VAS pain scale. Six patients reported positive change in mood, they felt much better in general mood after the treatment. One reported no change, and three patients reported negative change in mood, i.e., they felt worse in mood after the treatment. The group means, however changed little. The pre- 
treatment mean was 52.9 and the post-treatment mean 59.7. These mood results are consistent with prior findings on myofascial face pain patients. There we found that mood was stable over time (Zautra et al. 1995). Thus, we would expect that therapy directed at pain relief would not likely result in a rapid change in mood. In terms of the verbal description of pain, four patients reported no change, four reported reduction in pain, and two reported slight increase in pain, one of which is inconsistent with the visual analogue pain scale report.

To determine whether the results were due to a bias regarding belief in alternative therapy, we examined the associations between belief in alternative medicine and change in pain and mood. The correlation between belief and pain was modest, $r=0.174$, and for belief and mood negative, $r=-0.448$. These counterintuitive findings argue against a positive expectancy effect accounting for our results.

Conclusion: It is generally known that qigong practice is beneficial to human health and can prevent disease; however, it is less known, even in China, that qigong may be an effective therapy to treat various diseases, including chronic pain. Although the results of this study may be far from conclusive due to the small sample without a control treatment, the data indicate that, like many treatment experiences, Qigong therapy has some effect on pain-relief for most of our participants. The positive response we observed from most of our patients does suggest that it deserves further pursuit in the field with better design and larger sample. The possibility of a placebo or a regression-toward-mean effect accounting for the results cannot be excluded in such an open trial. 


\section{References:}

Eisenberg DM, Davis RB, Ettner SL, Appel S, Wilkey S, Van Rompay M, Kessler RC. Trends in alternative medicine use in the United States, 1990-1997 results of a follow-up national survey. JAMA. 1998; 280:1569-1575.

Wallenstein SL, Heidrich III G, Kaiko R, Houde RW. Clinical evaluation of mild analgesics: the measurement of clinical pain. Br. J. Clin. Pharmac. 1980;10:319-327S.

Zautra AJ, Marbach JJ, Raphael KG, Dohrenwend BP, Lennon MC, Kenny DA. The Examination of Myofascial Face Pain and Its Relationship to Psychological Distress in Women. Health Psychology 1995;14:223-231.

Kevin Chen, Ph.D., M.P.H.

Assistant Professor of Psychiatry

Joseph J. Marbach, D.D.S.

Robert and Susan Carmel Professor in Algesiology

University of Medicine and Dentistry of New Jersey

Robert Wood Johnson Medical School,

671 Hoes Lane. UBHC-D453,

Piscataway, NJ 08854 (USA).

Tel: 732-235-4345; fax: 732-235-5818;

Email: chenke@umdnj.edu 
Table 1

Visual Analogue Scale Pain Score Before and after the Qigong Intervention

\begin{tabular}{c|c|c|c|c}
\hline Case ID & Before & After & Change & $\%$ of Change \\
\hline 1 & 22 & 43 & +21 & $+95.0 \%$ \\
\hline 2 & 78 & 69 & -9 & $-11.5 \%$ \\
\hline 3 & 71 & 51 & -20 & $-28.2 \%$ \\
\hline 4 & 49 & 34 & -15 & $-30.6 \%$ \\
\hline 5 & 4 & 2 & -2 & $-50.0 \%$ \\
\hline 6 & 87 & 67 & -20 & $-23.0 \%$ \\
\hline 7 & 53 & 13 & -40 & $-75.5 \%$ \\
\hline 8 & 21 & 17 & -4 & $-19.0 \%$ \\
\hline 9 & 30 & 9 & -21 & $-70.0 \%$ \\
\hline 10 & 60 & 60 & 0 & $0.0 \%$ \\
\hline Means & 47.5 & 36.5 & -11.0 & $-23.2 \%$ \\
\hline
\end{tabular}

